\title{
Diabete post-trapianto renale
}

\author{
M. Parrini ${ }^{1}$, D. Angelini ${ }^{1}$, G. Penno ${ }^{2}$, A. Rosati ${ }^{1}$ \\ ${ }^{1}$ U.O Nefrologia, Ospedale Campo di Marte, Lucca \\ ${ }^{2}$ Azienda Ospedaliera Universitaria Pisana, Pisa
}

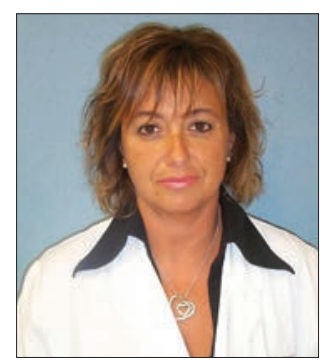

Manuela Parrini dell'iperglicemia è fondamentale per ottimizzare la terapia del post-trapianto.

Il DMPT è una forma di diabete mellito tipo II (3). L'iperglicemia risulta quindi determinata dallo squilibrio fra la produzione di insulina e la richiesta da parte degli organi bersaglio. In particolare, nel post-trapianto lo squilibrio può avvenire per l'insulino resistenza, per l'aumentato metabolismo dell'insulina, per una ridotta secrezione di insulina o più verosimilmente per una combinazione di tali meccanismi. L'insorgenza di DMPT è un evento piuttosto comune; $\mathrm{i}$ registri statunitensi riportano una incidenza di circa il 20\% a un anno dal trapianto (4), dati simili sono stati riportati in Francia in uno studio osservazionale multicentrico (5).

\section{Fattori di rischio}

I pazienti trapiantati sono particolarmente suscettibili a sviluppare il diabete per l'intervenire di numerosi fattori di rischio aggiuntivi rispetto a quelli presenti nella popolazione generale come il trattamento con agenti immunosoppressori utilizzati in tutti i protocolli terapeutici. Alcuni di questi fattori non sono modificabili come l'età avanzata, il sesso maschile, la suscettibilità genetica, altri come la presenza nella fase precedente al trapianto di sindrome metabolica, di obesità, di elevati livelli di trigliceridi, di infezione da citomegalovirus, di epatite $\mathrm{HCV}$ correlata sono invece modificabili (1); attenzione parti- colare merita la scelta della terapia immunosoppressiva tra cui l'esposizione ai corticosteroidi e l'uso di inibitori della calcineurina (6).

La terapia steroidea è stata e rimane un importante componente sia dell'induzione che del mantenimento dellimmunosoppressione ma gli effetti dello steroide sul metabolismo glucidico sono conosciuti già dal 1950 e l'associazione tra la terapia corticosteroidea e lo sviluppo di diabete dopo trapianto veniva segnalata per la prima volta già nel 1970 (7). L'effetto diabetogeno dello steroide è probabilmente dose correlato. Hjelmesaeth et al hanno evidenziato che l'aumento della dose di prednisolone di $0,01 \mathrm{mg} / \mathrm{kg}$ al giorno è associato all'aumento del 5\% del rischio di sviluppare DMPT (8). Alcuni autori hanno dimostrato che in pazienti con trapianto renale la sospensione del prednisolone è associata al miglioramento del metabolismo glucidico (9), mentre altri studi suggeriscono che la riduzione del dosaggio di prednisolone al di sotto di $5 \mathrm{mg} /$ die non apporta ulteriori benefici (10). In uno studio multicentrico, prospettico randomizzato in doppio cieco, Woodle et al (11) hanno confrontato, in un follow-up di cinque anni l'outcome tra due gruppi di pazienti con trapianto renale: 191 pazienti sospendevano precocemente la terapia corticosteroidea, mentre 195 pazienti ricevevano basse dosi di prednisone: tra i due gruppi non sono state registrate differenze significative per quanto riguarda l'outcome primario (outcome composito di morte, perdita dell'organo trapiantato o comparsa di rigetto acuto moderato/ severo). Tra i due gruppi non vi erano differenze neppure nell'incidenza di DMPT sebbene pochissimi pazienti che avevano sospeso il cortisone necessitassero di terapia insulinica. In conclusione, anche se il rischio di sviluppare DMPT dopo terapia con basse dosi di corticosteroide è molto basso, sembra comunque consigliabile la precoce sospensione dello steroide in quei pazienti ad alto rischio di sviluppare diabete già prima del trapianto. Anche gli inibitori della calcineurina esercitano un ef- 
fetto diabetogeno. L'analisi di Kasiske et al (4) ha evidenziato che l'incidenza di DMPT è più elevata nei pazienti trattati con Tacrolimus rispetto a quelli non trattati (13,5\% verso $7 \%$ a 3 mesi, $22,1 \%$ verso $14,2 \%$ a 12 mesi e $31,8 \%$ verso $21,9 \%$ a 36 mesi). Anche dopo correzione per altri fattori, il rischio relativo di sviluppare DMPT era significativamente più elevato nei soggetti trattati con Tacrolimus (rischio relativo 1,53, $\mathrm{p}<0,0001$ ). Woodward et al, analizzando i pazienti sottoposti a trapianto renale afferenti al database del USRDS, hanno osservato che a due anni dal trapianto l'incidenza di DMPT era del $70 \%$ più alta nei pazienti in trattamento con Tacrolimus rispetto a quelli in terapia con Ciclosporina (29,7\% verso $17,9 \%)$. Inoltre, nei soggetti in trattamento con Tacrolimus il rischio di diabete continuava ad aumentare anche dopo due anni dal momento del trapianto mentre nei pazienti in terapia con Ciclosporina tale rischio si riduceva. Si concludeva pertanto che sia il Tacrolimus che la Ciclosporina conferiscono un aumentato rischio di sviluppare DMPT ma che il Tacrolimus ha un potere diabetogeno maggiore rispetto alla Ciclosporina (12). Questi risultati sono stati confermati dallo studio DIRECT (13) che ha indagato l'incidenza di end point compositi di sicurezza (incidenza di DMPT o ridotta tolleranza glucidica) e di efficacia (rigetto acuto dimostrato da biopsia renale, perdita del trapianto o morte) in pazienti con trapianto renale de novo randomizzati al trattamento con Tacrolimus ( $n=346)$ o Ciclosporina ( $n=336)$; tutti i pazienti ricevevano micofenolato mofetil, corticosteroidi e basiliximab. Dopo sei mesi, l'incidenza di end point di sicurezza era significativamente maggiore nel gruppo trattato con Tacrolimus rispetto a quello trattato con Ciclosporina $(33,6 \%$ versus $26 \%, \mathrm{p}=0,046)$ mentre non si sono evidenziate differenze significative per quanto riguarda l'efficacia. Anche questo studio conferma l'ipotesi che il Tacrolimus sia più diabetogeno rispetto alla Ciclosporina. D'altra parte un altro studio non ha trovato differenze significative sul rischio di incorrere in alterazioni del metabolismo glucidico in pazienti che venivano convertiti tardivamente da Ciclosporina a Tacrolimus rispetto a quelli che continuavano la Ciclosporina (14). Anche il Sirolimus è stato identificato come farmaco diabetogeno. Johnston et al (15) analizzando i dati del USRDS hanno osservato che la Rapamicina, indipendentemente dalla terapia con la quale è in combinazione (inibitori della calcineurina o con antimetaboliti come micofenolato o azatioprina), è associata a un aumentato rischio di DMPT. In uno studio prospettico su pazienti con trapianto renale, Teutonico et al hanno dimostrato che la conversione della terapia da inibitori della calcineurina a Sirolimus si associava a una riduzione della sensibilità all'insulina; questa alterazione correlava con un aumento dei livelli di trigliceridi, effetto collaterale comune della terapia con gli inibitori della mTOR. Non è tuttavia noto se l'inibizione della mTOR sia associata alla comparsa di DMPT anche quando l'ipertrigliceridemia è appropriatamente controllata dalla terapia (16).

\section{Diagnosi e trattamento}

Studi epidemiologici indicano che annualmente circa il $6 \%$ dei pazienti candidati al trapianto renale sviluppa diabete (12). Questa prevalenza aumenta di circa due-tre volte nel primo anno dopo il trapianto e ritorna ai livelli basali molti anni dopo. Per questo motivo è fondamentale un'attento screening del diabete, mentre il trattamento deve iniziare già nella valutazione pre-trapianto $\mathrm{e}$ continuare in tutto il periodo successivo.

\section{Screening pre-trapianto}

Alla visita pre-trapianto, oltre alla raccolta dell'anamnesi e della storia familiare, deve essere attentamente valutata la presenza di fattori di rischio. Il dosaggio della glicemia a digiuno (FPG) e la valutazione della tolleranza glucidica (OGTT, oral glucose tolerance test) devono essere eseguiti nel contesto della visita di screening. FPG dovrebbe essere valutata alla prima visita e successivamente a cadenze regolari. Bloom et al (17) raccomandano di eseguire l'OGTT anche nei pazienti con glicemia a digiuno normale per diversi motivi: 1) individuare i soggetti con iperglicemia postprandiale isolata; 2) identificare i pazienti normoglicemici ma con storia preesistente di diabete mellito mascherato dal ridotto metabolismo dell'insulina come si verifica nell'insufficienza renale; 3 ) valori più alti di glicemia alla $2^{\text {a }}$ ora sono predittivi di DMPT e possono inoltre riflettere un aumento del rischio cardiovascolare. Sebbene non sia mai stata definita la frequenza con la quale eseguire l'OGTT, considerando che l'incidenza annuale di diabete è pari a circa il $6 \%$, sembra ragionevole ripetere il test ogni 1-2 anni. Se l'OGTT pone diagnosi di diabete mellito, il paziente deve iniziare la terapia, se si evidenziano invece IFG/IGT al paziente dovranno essere raccomandate appropriate modificazioni dello stile di vita. Infine si dovrebbe eseguire anche lo screening (ed eventuale trattamento) per altri due fattori di rischio come la sindrome metabolica che è particolarmente comune nei soggetti uremici e la cui identificazione permette di intervenire in maniera appropriata e aggressiva sui fattori di rischio per diabete e l'infezione da HCV. In questo caso, la terapia con interferon potrebbe annullare la viremia, condizione fondamentale per ridurre il rischio di DMPT (17) (Tab. I). 
TABELLA I - SCREENING E TRATTAMENTO

\begin{tabular}{|c|c|c|c|}
\hline Parametro & Prima del trapianto & Perioperatorio & Dopo la dimissione \\
\hline Screening & $\begin{array}{l}\text { OGTT } 75 \text { gr ogni } 1-2 \text { anni } \\
\text { Screening per sindrome metabolica ogni anno } \\
\text { Test per HCV ogni anno }\end{array}$ & $\begin{array}{l}\text { Stick glicemici } \\
\text { multipli pre- e post- } \\
\text { prandiali }\end{array}$ & $\begin{array}{l}\text { FPG/settimana } \\
\text { nel primo mese } \\
\text { FPG ogni } 4 \text { settimane } \\
\text { nei primi sei mesi } \\
\text { FPG ogni } 8 \text { settimane } \\
\text { nel primo anno } \\
\text { OGTT } 75 \text { gr a tre e sei mesi } \\
\text { OGTT annualmente } \\
\text { dopo il primo anno }\end{array}$ \\
\hline Trattamento & & 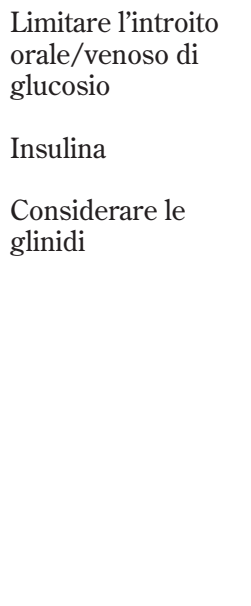 & $\begin{array}{l}\text { Terapia nutrizionale } \\
\text { Attività fisica moderata } \\
\text { Perdita di peso } \\
\text { Ottimizzare la terapia } \\
\text { immunosoppressiva } \\
\text { Insulina/ antidiabetici } \\
\text { orali come: } \\
\text { TZDs } \\
\text { Glinidi } \\
\text { Sulfoniluree } \\
\text { Exenatide, Liraglutide } \\
\text { Inibitori DPP-IV } \\
\text { Trattamento } \\
\text { ipolipemizzante }\end{array}$ \\
\hline
\end{tabular}

Modificata da Bloom R.D, Clin J Am Soc Nephrol 2008, 3: S38-S48.

\section{Valutazione e terapia nell'immediato post-trapianto}

L’iperglicemia è un evento comune nellimmediato posttrapianto tipicamente in relazione allo stress chirurgico e agli alti dosaggi di glucocorticoidi che si risolve subito dopo la riduzione della terapia con lo steroide; se l'iperglicemia persiste oltre una settimana è predittiva di alterazione glucidica a lungo termine (18).

Lo stick glicemico pre- e post-prandiale dovrebbe essere eseguito più volte al giorno fino a che il dosaggio dello steroide non è inferiore a $20 \mathrm{mg} /$ die e fino alla normalizzazione della glicemia sia a digiuno che dopo i pasti. Secondo i criteri dell'American Diabetes Association (ADA) i livelli glicemici per i pazienti ancora ospedalizzati dovrebbero essere fra 90 e $130 \mathrm{mg} / \mathrm{dL}$ a digiuno e inferiori a $180 \mathrm{mg} / \mathrm{dL}$ dopo i pasti.

L'approccio terapeutico iniziale non può prescindere da un adeguato controllo dietetico limitando l'apporto di carboidrati a 130-180 gr al giorno con un introito calorico totale non superiore a 1800-2000 calorie. Se persiste l'iperglicemia il trattamento con insulina è l'unico trattamento possibile nell'immediato post-trapianto (Tab. I).

\section{Valutazione nel lungo termine}

Lo screening periodico per la presenza di diabete è fondamentale anche nei pazienti che alla dimissione presentavano valori di glicemia nella norma anche se l'entità della sorveglianza tende a declinare nel tempo (Tab I). Le linee guida internazionali hanno proposto una valutazione settimanale nel primo mese dal trapianto e successivamente ogni tre mesi (19). Nei pazienti ad alto rischio di sviluppare il DMPT viene raccomandato uno screening più aggressivo: FPG settimanalmente nel primo mese, una volta al mese nei successivi sei mesi, ogni due mesi fra 6 e 12 mesi. L'esecuzione di OGTT viene proposta a tre e a sei mesi dopo il trapianto e successivamente annualmente. Se viene diagnosticato DMPT il trattamento dovrebbe essere iniziato secondo le indicazioni dell'American 
TABELLA II - TERAPIA NON INSULINICA

\begin{tabular}{|c|c|c|c|c|c|c|}
\hline Classe & $\begin{array}{l}\text { Meccanismo } \\
\text { di azione }\end{array}$ & Esempio & $\begin{array}{l}\text { Effetti } \\
\text { su HbA1c (\%) }\end{array}$ & $\begin{array}{l}\text { Eventi } \\
\text { avversi }\end{array}$ & $\begin{array}{l}\text { Metabolismo/ } \\
\text { eliminazione }\end{array}$ & $\begin{array}{l}\text { Aggiustamento } \\
\text { dosaggio in IRC }\end{array}$ \\
\hline Sulfoniluree & $\begin{array}{l}\text { Stimolazione } \\
\text { della secrezione } \\
\text { di insulina }\end{array}$ & $\begin{array}{l}\text { Glipizide } \\
\text { Gliburide } \\
\text { Glimepiride }\end{array}$ & $-1 /-2$ & Ipoglicemia & $\begin{array}{l}\text { Maggiore: } \\
\text { epatico }\end{array}$ & $\begin{array}{l}\text { Sì (in relazione } \\
\text { rischio } \\
\text { ipoglicemia) }\end{array}$ \\
\hline Glinidi & $\begin{array}{l}\text { Stimolazione } \\
\text { della secrezione } \\
\text { di insulina }\end{array}$ & $\begin{array}{l}\text { Repaglinide } \\
\text { Nateglinide }\end{array}$ & -0.6 & Ipoglicemia & $\begin{array}{l}\text { Maggiore: } \\
\text { CYP2C8 e } \\
3 \mathrm{~A} 4\end{array}$ & $\begin{array}{l}\text { Cl.creat } \\
>40 \mathrm{ml} / \mathrm{min}: \mathrm{NO} \\
\text { Cl.creat }<40 \mathrm{ml} / \mathrm{min} \\
\text { Introduzione } \\
\text { graduale }\end{array}$ \\
\hline Biguanidi & $\begin{array}{l}\text { Riduzione } \\
\text { della produzione } \\
\text { epatica di glucosio }\end{array}$ & Metformina & $-1 /-1.7$ & $\begin{array}{l}\text { Nausea } \\
\text { Acidosi lattica }\end{array}$ & $\begin{array}{l}\text { Maggiore: } \\
\text { renale, } \\
\text { secrezione } \\
\text { tubulare }\end{array}$ & $\begin{array}{l}\text { Evitare con IRC } \\
\text { (stadi } 3 \text { e } 4 \text { ) }\end{array}$ \\
\hline TZD & $\begin{array}{l}\text { Aumento } \\
\text { della sensibilità } \\
\text { all'insulina }\end{array}$ & Pioglitazone & $-1 /-1.9$ & $\begin{array}{l}\text { Aumento } \\
\text { di peso } \\
\text { Ritenzione } \\
\text { idrica }\end{array}$ & $\begin{array}{l}\text { Maggiore: } \\
\text { CYP2C8 } \\
\text { Minore: } \\
\text { CYP3A4 }\end{array}$ & NO \\
\hline GLP-1 agonista & $\begin{array}{l}\begin{array}{l}\text { Stimolazione } \\
\text { della secrezione } \\
\text { di insulina }\end{array} \\
\text { Inibizione } \\
\text { del Glucagone } \\
\text { Induce perdita } \\
\text { di peso tramite } \\
\text { il rallentamento } \\
\text { dello svuotamento } \\
\text { gastrico } \\
\text { e della riduzione } \\
\text { dell'appetito }\end{array}$ & $\begin{array}{l}\text { Exenatide } \\
\text { Liraglutide }\end{array}$ & $-0.4 /-0.8$ & $\begin{array}{l}\text { Nausea } \\
\text { e disturbi } \\
\text { gastro- } \\
\text { intestinali }\end{array}$ & Maggiore: renale & $\begin{array}{l}\text { Da evitare } \\
\text { se creat }>2 \mathrm{mg} / \mathrm{dL}\end{array}$ \\
\hline DPP-IV & $\begin{array}{l}\text { Aumento } \\
\text { del GLP-1 }\end{array}$ & $\begin{array}{l}\text { Sitagliptina } \\
\text { Vildagliptina } \\
\text { Saxagliptin }\end{array}$ & -0.5 & Minimi & $\begin{array}{l}\text { Maggiore: } \\
\text { renale } \\
\text { Minore: } \\
\text { CYP2C8 } \\
\text { e } 3 \mathrm{~A} 4\end{array}$ & $\begin{array}{l}\text { Riduzione } \\
\text { del dosaggio }\end{array}$ \\
\hline
\end{tabular}

Modificata da Bloom R.D, Clin J Am Soc Nephrol 2008 ; 3: S38-S48.

Diabetes Association che ha definito i seguenti target: FPG tra 90 e $130 \mathrm{mg} / \mathrm{dL}$, e due ore dopo i pasti $<180$ $\mathrm{mg} / \mathrm{dL}$; l'emoglobina glicosilata (HbA1c) dovrebbe essere inferiore a $7 \%(20)$.

Il trattamento a lungo termine del DMPT è multifattoriale e prevede sia l'intervento sullo stile di vita sia modificazioni della terapia immunosoppressiva e terapia specifica.

\section{Trattamento farmacologico}

Le opzioni terapeutiche per il trattamento del diabete sono in continua espansione sebbene i dati sulla sicurezza e l'efficacia della maggior parte degli agenti ipoglicemizzanti siano limitati nei pazienti portatori di trapianto. Molti farmaci ipoglicemizzanti possono avere interazioni con gli agenti immunosoppressori sia agendo sul loro 
metabolismo che modificando la motilità gastrica. Generalmente la combinazione di più agenti ipoglicemizzanti diversi è più efficace della monoterapia; le sulfoniluree e le glinidi per il loro meccanismo di azione non possono essere associate fra loro (Tab. II).

\section{Insulina}

I regimi terapeutici in monosomministrazione con le nuove insuline long-acting come la Glargina e la Detemir provvedono al controllo glicemico basale, mentre gli analoghi dell'insulina rapida come Aspart, Lispro e Glulisina provvedono al controllo glicemico post-prandiale. Queste nuove insuline sono preferibili a quelle tradizionali perché permettono di raggiungere il target glicemico più facilmente e con minor rischio di eventi ipoglicemici. Poiché il rene gioca un ruolo importante nel metabolismo dell'insulina, disfunzioni dell'organo trapiantato possono rendere necessarie frequenti ed accurate variazioni del dosaggio. Linsulina non sembra interferire sul metabolismo degli agenti immunosoppressori (17).

\section{Sulfoniluree}

Le sulfoniluree stimolano direttamente la secrezione insulinica e riducono sia la glicemia basale che quella post-prandiale. Le sulfoniluree di seconda generazione (Glipizide, Gliburide, Glimepiride) sono in pratica le uniche ad essere utilizzate. La Glipizide è preferibile in caso di alterazioni della funzione renale perché viene metabolizzata interamente dal fegato. Essa rappresenta una buona opzione terapeutica in caso di iperglicemia non severa, può essere introdotta a basso dosaggio e titolata ogni 3-4 settimane. Poiché il principale effetto collaterale delle sulfoniluree è l'ipoglicemia questi farmaci devono essere usati con molta precauzione in caso di riduzione della funzione renale (17).

\section{Glinidi}

La Repaglinide e la Nateglinide sono secretagoghi dell'insulina con una rapida e breve durata di azione; possono essere assunti immediatamente prima dei pasti e presentano minor frequenza di episodi di ipoglicemia rispetto alle sulfoniluree. Sono metabolizzate dal fegato e possono essere usate con ogni livello di funzione renale (17).

\section{Tiazolidinedioni (TZDs)}

TZDs promuovono la sensibilità periferica all'insulina e sono usati ampiamente nel trattamento del diabete di tipo 2. Il Pioglitazone, l'unico TZD attualmente disponi- bile, ha mostrato una modesta efficacia nella prevenzione di eventi cardiovascolari in soggetti con diabete e può essere somministrato anche in soggetti con riduzione della funzione renale. Il Rosiglitazone è stato recentemente ritirato perché il trattamento è stato associato ad un eccesso di eventi cardiovascolari (17).

\section{Glucagon-Like Peptide-1 (GLP-1) mimetici e GLP-1 enhancers}

GLP-1 è un ormone prodotto a livello intestinale che stimola la secrezione di insulina dalle cellule beta delle isole pancreatiche in maniera glucosio-dipendente. GLP-1 rallenta lo svuotamento gastrico e controlla l'appetito con sostanziali effetti benefici sul peso corporeo. L'Exenatide e l'Iraglutide sono gli analoghi del GLP-1 attualmente disponibili per l'uso clinico. La prima viene usata due volte al giorno per via iniettiva prima dei pasti, controlla il picco glicemico post-prandiale e determina una riduzione stabile dell'HbA1c. La seconda viene usata in monosomministrazione giornaliera. Al momento però non sono riportati dati sull'utilizzo degli analoghi del GLP-1 nei pazienti con trapianto renale.

Il GLP-1 viene degradato dalla dipeptidyl peptidasi IV (DPP-IV). Sitagliptina, Saxagliptin e Vildagliptina sono state recentemente approvate per l'uso clinico. Sono inibitori della DPP-IV per cui aumentano le concentrazioni endogene del GLP-1 attivo, riducono il picco glicemico postprandiale e riducono i livelli di HbA1c dallo 0,6 allo 0,8\%. Sono comunque disponibili pochi dati sull'interazione con i farmaci immunosoppressori per cui il loro uso richiede un attento monitoraggio dei loro livelli ematici (17).

\section{Biguanidi}

La Metformina riduce la produzione epatica di glucosio ed è generalmente efficace sia nel controllo dell'iperglicemia sia nella riduzione e nella stabilizzazione del peso corporeo. Viene metabolizzata a livello renale con un'azione di secrezione tubulare inoltre, potendo indurre un'acidosi lattica, il suo uso è assolutamente sconsigliato in corso di insufficienza renale (17).

\section{Conclusioni}

Il DMPT determina un significativo aumento della morbilità e mortalità dei pazienti sottoposti a trapianto renale. L'individuazione e il trattamento, dove possibile, dei fattori di rischio modificabili sono step fondamentali per minimizzare tali complicanze. Una stretta valutazione dei soggetti ad alto rischio è importante già nella fase di pre-trapianto e lo screening nel post-trapianto è indicato 
per tutti i pazienti. Modificare i fattori di rischio conosciuti per lo sviluppo di DMPT e di patologie cardiovascolari offre la miglior opzione per prolungare la vita del trapianto e del paziente. Tra i fattori di rischio per lo sviluppo di DMPT (età, razza/etnia, sieropositività per l'epatite C, storia familiare per diabete tipo II, sovrappeso e terapia immunosoppressiva) solo gli ultimi due sono efficacemente modificabili. Lintervento sullo stile di vita dovrebbe promuovere la riduzione dell'introito di carboidrati, l'aumento dell'attività fisica in tutti i pazienti e la riduzione del peso corporeo in quelli sovrappeso. La scelta del regime immunosoppressivo non può prescindere dalla ricerca di fattori di rischio nello screening pre-trapianto. Attualmente sono disponibili numerosi agenti farmacologici per raggiungere il target glicemico; molti di questi farmaci richiedono un aggiustamento del dosaggio in caso di disfunzione renale e molti di loro possono interferire con la terapia immunosoppressiva. E auspicabile un approccio multifattoriale per ottimizzare il controllo glicemico e il buon funzionamento del trapianto.

\section{Indirizzo degli Autori:}

Manuela Parrini, MD

U.O. Nefrologia Ospedale Campo di Marte

Via dell'Ospedale 1

55100 Lucca

m.parrini@usl2.toscana.it

\section{Bibliografia}

1. Sharif A, Baboolal K. Risk factors for new-onset diabetes after kidney transplantation. Nat Rev Nephrol 2010; 6: 415-23.

2. Burroughs T.E, et al. Diabetic complications associated with new-onset diabetes mellitus after transplant recipients. Transplantation 2007; 83:1027-34.

3. Van Hooff JR, Christiaans MH, van Duijnhoven EM. Evaluating mechanisms of post-transplant diabetes mellitus. Nephrol Dial Transplant 2004; 19 (Suppl 6): vi8-vi12.

4. Kasiske B, Snyder JJ, Gilberston D, et al. Diabetes mellitus after Kidney transplantation in the United States. Am J Transplant 2003; 3:178-85.

5. Kamar N, Mariat C, Delahousse M, et al. Diabetes mellitus after Kidney transplantation: a French multicentre observational study. Nephrol Dial Transplant 2007; 22: 1986-93.

6. Hjelmesaeth J, Midtved K, Jenssen T, et al. Insulin resistance after renal transplantation: impact of immunosuppressive and antihypertensive therapy. Diabetes Care 2001; 24: 2121-6.
7. Hill CM, Douglas JF, Rajkumar KV, et al. Glycosuria and hyperglycaemia after kidney transplantation. Lancet 1974; 2: 490-2.

8. Hjelmesaeth J, Hartmann A, Kofstad J, et al. Tapering off prednisolone and cyclosporin the first year after renal transplantation: the effect on glucose tolerance. Nephrol Dial Transplant 2001; 16: 829-35.

9. Boots JM, van Duijnhoven EM, Christiaans MH, et al. Glucose metabolism in renal transplant recipients on tacrolimus: the effect of steroid withdrawal and tacrolimus trough level reduction. J Am Soc Nephrol 2002; 13: 221-7.

10. Midtvedt K, Hjelmesaeth J, Hartmann A, et al. Insulin resistance after renal transplantation: the effect of steroid dose reduction and withdrawal. J Am Soc Nephrol 2004; 15: 3233-9.

11. Woodle ES, First MR, Pirsch J, et al. A prospective, randomized, double blind, placebo controlled multicenter trial comparing early (7 day) corticosteroid cessation versus long term, low dose corticosteroid therapy. Ann Surg 2008; 248: 564-77.

12. Woodward RS, Schnitzler MA, Baty J, et al. Incidence and cost of new onset diabetes mellitus among U.S wait-listed and transplanted renal allograft recipients. Am J Transplant 2003; 3: 590-8.

13. Vincenti F, Friman S, Scheuermann E, et al. Results of an international, randomized trial comparing glucose metabolism disorders and outcome with cyclosporine versus tacrolimus. Am J Transplant 2007; 7: 1506-14.

14. Luan FL, Zhang H, Schaubel DE, et al. Comparative risk of impaired glucose metabolism associated with cyclosporine versus tacrolimus in the late posttransplant period. Am J Transplant 2008; 8: 1871-7.

15. Johnston O, Rose CL, Webster AC, et al. Sirolimus is associated with new-onset diabetes in Kidney transplant recipients. J Am Soc Nephrol 2008; 19: 1411-8.

16. Teutonico A, Schena PF, Di Paolo S. Glucose metabolism in renal transplant recipients: effect of calcineurin inhibitor withdrawal and conversion to sirolimus. J Am Soc Nephrol 2005; 16: 3128-35.

17. Bloom RD, Crutchlow MF. New-Onset diabetes mellitus in the kidney recipient: diagnosis and management strategies. Clin J Am Soc Nephrol 2008; 3(Suppl): S38-S48.

18. Cosio FG, Kudva $Y$, van der Velde $M$ et al. New onset hyperglycemia and diabetes are associated with increased cardiovascular risk after kidney transplantation. Kidney Int 2005; 67: 2415-21.

19. Wilkinson A, Davidson J, Dotta F, et al. Guidelines for the treatment and management of new-onset diabetes after transplantation. Clin Transplant 2005; 19: 291-8.

20. American Diabetes Association: Standards of medical care in diabetes-2006. Diabetes Care 2006; 29 (Suppl 1): S4-S42. 